\title{
Methods for Evaluation of Imperfect Captioning Tools by Deaf or Hard-of-Hearing Users at Different Reading Literacy Levels
}

\author{
Larwan Berke \\ Rochester Institute of Technology \\ Rochester, NY, USA \\ larwan.berke@mail.rit.edu
}

\author{
Sushant Kafle \\ Rochester Institute of Technology \\ Rochester, NY, USA \\ sushant@mail.rit.edu
}

\author{
Matt Huenerfauth \\ Rochester Institute of Technology \\ Rochester, NY, USA \\ matt.huenerfauth@rit.edu
}

\begin{abstract}
As Automatic Speech Recognition (ASR) improves in accuracy, it may become useful for transcribing spoken text in real-time for Deaf and Hard-of-Hearing (DHH) individuals. To quantify users' comprehension and opinion of automatic captions, which inevitably contain some errors, we must identify appropriate methodologies for evaluation studies with DHH users, including quantitative measurement instruments suitable to the various literacy levels among the DHH population. A literature review guided our selection of several probes (e.g. multiple-choice comprehension-question accuracy or response time, scalar-questions about user estimation of ASR errors or their impact, users' numerical estimation of accuracy), which we evaluated in a lab study with DHH users, wherein their literacy levels and the actual accuracy of each caption stimulus were factors. For some probes, participants with lower literacy had more positive subjective responses overall, and, for participants with particular literacy score ranges, some probes were insufficiently sensitive to distinguish between caption accuracy levels.
\end{abstract}

\section{Author Keywords}

Accessibility; Captioning; People who are Deaf or Hard-ofHearing; Literacy; Evaluation Methods

\section{ACM Classification Keywords}

- Human-centered computing Empirical studies in accessibility - Human-centered computing Accessibility design and evaluation methods

\section{INTRODUCTION}

People who are Deaf and Hard of Hearing (DHH) make use of a wide variety of communication technologies and accommodations, e.g. real-time captioning services produced by professional transcriptionists (with text displayed on a screen for the user) or American Sign Language (ASL) interpreting $[3,26]$. While laws in some countries require the

\footnotetext{
Permission to make digital or hard copies of all or part of this work for personal or classroom use is granted without fee provided that copies are not made or distributed for profit or commercial advantage and that copies bear this notice and the full citation on the first page. Copyrights for components of this work owned by others than ACM must be honored. Abstracting with credit is permitted. To copy otherwise, or republish, to post on servers or to redistribute to lists, requires prior specific permission and/or a fee. Request permissions from Permissions@acm.org.
}

CHI 2018, April 21-26, 2018, Montreal, QC, Canada

(C) 2018 Association for Computing Machinery.

ACM ISBN 978-1-4503-5620-6/18/04 _.\$15.00

https://doi.org/10.1145/3173574.3173665 provision of such services in educational settings, in many workplace settings that require real-time face-to-face communication, DHH individuals may not have access, e.g. due to cost, insufficient service providers, or the need to schedule services in advance. DHH users may benefit from recent advances in Automatic Speech Recognition (ASR), which could transcribe speech to text, and as mobile technologies proliferate, we can imagine a future where those devices, in conjunction with cloud-based ASR engines, become a useful communication tool for DHH individuals. In live meetings with hearing colleagues, users may view captions, e.g. as visualized in the prototype in Figure 1.

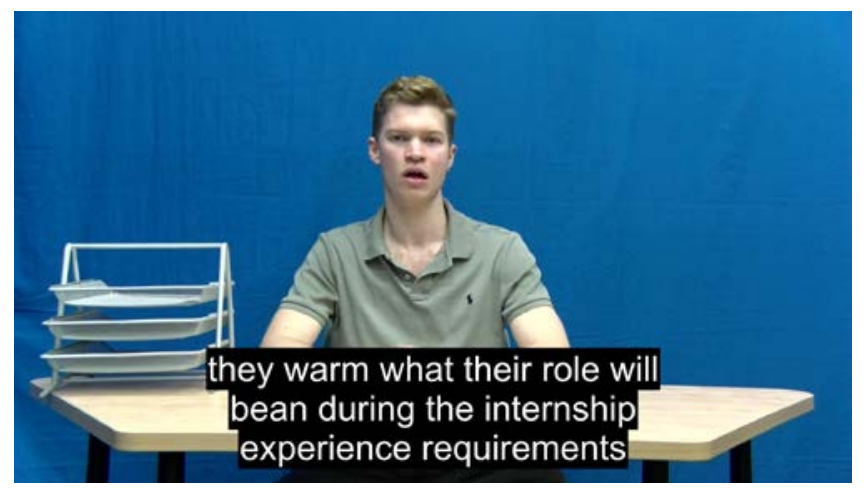

Figure 1. The research prototype tool examined in this work.

We discuss below how there is growing interest in using ASR to produce caption text automatically for DHH users, yet there are errors in ASR text output. Prior research with $\mathrm{DHH}$ users has found that they are concerned about this issue of accuracy. Creators of ASR-based systems for DHH users have a responsibility to evaluate their tools' usability, to ensure that the premature deployment of such tools does not replace higher-quality existing communication solutions. While it is possible to arrange empirical studies in which DHH users are provided with captioning that has been based on ASR, the challenge is that there is great variation in reading literacy skills among the DHH community, and there is prior evidence (discussed below) that users with lower literacy may be less critical when evaluating speech and language technologies, and they may be less able to discern quality differences in the imperfect output of these technologies. Unfortunately, studies of captioning system efficacy with DHH users rarely report the literacy skill level of participants, nor is this issue generally considered when measurement instruments are selected for studies. 
We have therefore conducted a study to evaluate the efficacy of a variety of measurement probes for gathering judgments about the quality and accuracy of captions, from DHH users. In this methodological study, we make the assumption that captions with more errors are worse, and we ask DHH users to view captions at three different quality levels. Participants have been grouped into three different reading literacy levels, and we are therefore able to examine how well each of the different types of probe can differentiate captions of different quality levels - and whether there may be a trend such that users with higher literacy give more critical/negative ratings.

The contributions of this study include: (a) methodological guidance in the form of an empirical comparison of the efficacy of different measurement probes for use in studies of captioning tools with DHH users of different literacy levels and (b) empirical evidence that researchers should report participants' reading literacy skills - so that results across studies with different user characteristics can be compared.

\section{Growing Interest and Risks of Premature Deployment}

As accessibility researchers investigate applying ASR to the task of captioning, many have focused on non-real-time contexts, e.g. after-the-fact transcripts of classroom lectures [42] or captioning online videos [38]. Some researchers have investigated semi-automated real-time systems (i.e. humans working in collaboration with ASR), e.g. ASR transcribing classroom lectures after humans prepare terminology lists for each course [21]. In some professional captioning services, a service provider uses a high-end microphone to clearly respeak the words of the lecturer for an ASR engine trained on the re-speaker's voice [28]. Some researchers have employed human "overseers" to fix errors in imperfect ASR output [11] or engaged humans to crowdsource the task of transcribing audio [13]. While those services have higher accuracy than current ASR technologies, the business model for those services requires some form of payment for the human labor.

While there has been recent interest from accessibility researchers in automated captioning systems for DHH users, ASR is imperfect: Although accuracy has been improving with advances in deep-learning techniques, ASR output still contains errors, especially in noisy realistic conversational speech settings. Despite this, researchers have investigated fully automated real-time applications of ASR, with some finding that DHH users are interested in the promise of ASR supporting their conversations $[2,7]$ but have concerns about accuracy when they actually try such systems [30]. In recent qualitative studies of DHH users' acceptance of ASR-based captioning technology, in classrooms [20] and in simulations of live meetings [2], researchers have found that users struggle with low-quality captions containing many errors. In fact, researchers argue that errors present in automatic ASRgenerated captions are even more comprehension-demanding than errors produced by human captioners [23].

As ASR improves, companies, governments, or universities may be interested in using ASR for captioning, but there is a risk that ASR could be deployed before the output is of acceptable accuracy. Some researchers have examined this issue from a legal lens [32], and many DHH users are concerned that automated systems will have lower accuracy than professional sign language interpreting or captioning, especially in critical settings like classrooms, courtrooms, doctor appointments, etc. This fear of replacing current services with a lower quality automated system has emerged in surveys of the accessibility literature, which found that users prefer "sign language interpretation in classes over other means of communication, and that acceptance of [ASR] systems is linked to the quality of transcriptions" [34]. As discussed above, researchers have a responsibility to measure the usability of such systems before deployment.

Prior Methods of Evaluating Captioning with DHH Users ASR researchers often employ intrinsic, automated metrics of evaluating their systems, typically by comparing the text output (from automatic recognition of speech) to a reference text (containing an accurate transcript of what was spoken). Although some have proposed alternative metrics [14], most researchers report accuracy using Word Error Rate (WER), which counts the number of discrepancies between these texts, divided by text length (more details in a later section). However, for the purpose of evaluating the efficacy of an ASR-based captioning system for use in meetings by DHH users, simply counting the number of errors in the output is too indirect a metric of success. While it is safe to assume, in general, that captioning systems that display more incorrect words are worse than systems displaying fewer, the specific levels of WER that DHH users will tolerate is unknown, and it likely depends on the specific conversation topic, the skill of particular users in coping with erroneous captions, etc.

For this reason, prior accessibility research on the usability of captioning technologies for DHH users utilizes extrinsic methods of evaluation, specifically metrics gathered through empirical evaluations of systems in studies with DHH users - see survey in [34]. Most studies have asked DHH users to provide subjective feedback on the system or answer comprehension questions, using a variety of question types:

- Open-ended questions to elicit feedback from users, e.g. "Were there any times that you thought about using the tool but decided not to or weren't able to?" [29]

- Scalar-response questions asking users for their subjective judgment of the system's usefulness [25], its understandability (e.g. "How easy was it to follow...") [22], or its caption accuracy as a percentage [4].

- Comprehension questions designed to measure users' understanding of information conveyed, through various question types: open-ended ("Who recommended the hot spring?") [15], fill-in-the-blank (“An unexpected negative outcome of industrialization was __.") [40], or multiple-choice ("What will the director of each team get? (a) a document; (b) discharged; (c) advice; (d) The information was not in the video clip." [2].

A complicating factor when selecting appropriate evaluation instruments for studies with DHH users is that there is great 
diversity among the DHH community in reading literacy skills. Some DHH adults have high levels of reading literacy, especially those who had early childhood access to language or high-quality educational experiences. Yet, approximately $30 \%$ of deaf students who graduate high school (secondary school) in the U.S. are "functionally illiterate" [27]. Even studies on deaf university students have found that most have reading levels below the sixth grade $[1,33]$ - U.S. students in the "sixth grade" are typically 11-12 years old.

\section{Conducting Evaluations with Users with Low Literacy}

Although not focused on DHH users, several prior studies have considered methodological issues in successfully gathering subjective feedback from users with low reading literacy skills. For instance, health researchers focused on people with low English fluency have argued that openended questions are ineffective with their users [6]. Others conducting surveys of adults with very low literacy have argued that observational studies of technology use can be more effective than asking users subjective questions [31].

Even more closely related to our study is prior work that has examined how participants with low-literacy can effectively evaluate the output of computational linguistic software that generates imperfect language, e.g. systems that are designed to automatically simplify complex texts for these users (but which may inadvertently introduce language errors in the process). These researchers have found that:

- Objective metrics, e.g. reading time or comprehension question scores, were ineffective at measuring text readability by adults with Down Syndrome [36], yet qualitative analysis of observations revealed that participants using the system had a positive experience and believed that it helped them read better.

- Yes/No questions and Likert-scale subjective questions were ineffective at evaluating text readability in studies with adults with intellectual disabilities [17].

- Analysis of eye-movements of readers with Dyslexia can be used to evaluate the readability of texts [35].

From this related work, we can see that designing effective studies to evaluate imperfect text output from language technology with users with low-literacy is not a trivial task. However, it is difficult to take specific guidance from this prior literature that can be applied to the design of studies in which DHH users evaluate ASR-based captioning systems: In addition to low reading literacy, users in [17, 36] experienced cognitive impairments that may have interfered with their ability to respond to evaluation questions. While researchers in [35] were able to model relationships between eye-movements and text readability, doing the same for users who are DHH is challenging: It is known that DHH readers have eye-movement patterns that differ from hearing readers [5], and users of a captioning system for meetings with hearing colleagues will divert their eye-gaze to their conversational partner at times, making it difficult to use eyemovements as a proxy for the readability of caption text.

\section{Studies with DHH Users with Low Language Fluency}

There is evidence that DHH individuals with lower language fluency have difficulty subjectively evaluating the quality of imperfect language output generated by software. In [18], DHH participants evaluated the quality of imperfect ASL animations generated by computational linguistic software; the output animations contained language errors. The researchers found that DHH individuals with lower language fluency (in this case, in ASL) gave overall higher scores to all animations they saw (they were less-critical judges), and they had difficulty distinguishing between ASL animations that the researchers knew were of different quality levels.

In this work, we investigate whether similar patterns hold true when asking DHH individuals with lower English fluency to evaluate the imperfect English text captions from ASR. If true, this raises several methodological concerns:

- If the literacy level of participants were to lead them to give overall higher or lower subjective scores to ASR captioning systems, then researchers should report the literacy level of the participants in their studies, which is currently not a common practice among researchers.

- If researchers wish to differentiate between whether their system is producing high- or low-quality captions, they must consider the literacy level of their participants, to select an appropriate question type capable of discriminating between caption quality levels.

- Since comprehension question scores are based on several factors, e.g. original text quality, the difficulty of the question, and the reading skill of the respondent such questions must be tailored to participants' literacy skills. E.g., participants with above-average fluency may better infer erroneous/missing information in captions.

\section{DESIGN OF OUR STUDY}

We conducted a study with DHH users to investigate whether there is a relationship between their literacy level and their responses to subjective or comprehension questions to evaluate a captioning system. Our literature review had not revealed prior methodological research that had employed various question types in a single study, to compare their efficacy at evaluating captioning systems with DHH users, especially in regard to the literacy level of the participants. Specifically, we asked $107 \mathrm{DHH}$ users to try a prototype ASR captioning system, in our case, presented as videos that simulate a one-on-one meeting with a hearing colleague in a workplace setting (Figure 1). After viewing the videos with captions, participants were asked a variety of question types (see list a-g below). Participants also completed a standard reading literacy test (WRAT, details below), and we grouped participants into three literacy levels, for analysis.

To determine whether various question types, for users of various literacy levels, were effective at distinguishing between high-quality and low-quality captions, we needed to include captions of various quality levels as stimuli in the study. We make the assumption that captions with more errors are worse than captions with fewer errors. We included 
captions at three levels of quality in the study, and we examined whether various question types could distinguish the three levels, for users of various literacy levels.

\section{Hypotheses and Question-Types in Our Study}

Our study investigated the following two hypotheses:

H1: If we focus on participants at a single literacy level, and we compare their response scores for captions of different quality levels, we will observe significant differences between their scores for captions at each quality level. We refer to this as the discriminative ability of the probe, and it is desirable for a question type to have this ability to distinguish caption quality.

H2: If we consider response scores for all captions in the study, and we compare the scores from participants at different literacy levels, we will observe significant differences in the responses from participants at each literacy level. We refer to this as the literacy bias of the probe; if researchers use question types with this bias but fail to control for or report participant literacy levels, then we cannot compare results across studies.

For each hypothesis, we examined seven sub-hypotheses, which correspond to each measurement probe (each type of question) used in the study. The first five require subjective judgments from participants, and the final two are more objective, relating to comprehension accuracy or speed.

a) Noticed errors in the captions (Boolean): After each video, participants responded Yes or No to the following: "Did you notice errors in the captions?"

b) Errors prevented understanding (Likert): After each video, we asked participants to indicate their agreement, on a 5-point Likert scale to the following: "The errors in the captioning prevented me from understanding what the speaker was saying."

c) ASR did a good job (Likert): After each video, we asked participants to indicate their agreement, on a 5point Likert scale to the following: "The Automatic Speech Recognition did a good job captioning what the speaker was saying."

d) Evaluation of accuracy (Ordinal Scale): After each video, participants were asked to use an ordinal scale of response options ("Zero, Low, Medium, High, or Perfect") to respond to: "The caption accuracy was."

e) Evaluation of accuracy (Numeric): After each video, participants were asked provide a number in response to this: "I would guess that the captioning accuracy is (0-100 where $100 \%$ means perfect)."

f) Comprehension quiz success (Multiple-Choice): After each video, participants answered four multiplechoice questions based on the information that had been spoken. Text-explicit (TE) style comprehension questions [41] were used, wherein the participant is not required to infer from information in memory. Each question had four answer choices: In random order, the list included a correct option and two incorrect options (containing words not present in the stimulus video). The final option was always "The information was not in the video clip," which was never the correct answer. The score for each video was the average of the scores for the four questions.

g) Comprehension quiz time (Seconds): The software used to present the questions recorded the total time participants spent reading the questions and selecting their answer choice, before submitting their response.

Our set of question types were inspired by prior studies in our literature review. Several questions were modeled after the Quality of Perception (QoP) scale shown to be effective for $\mathrm{DHH}$ individuals in [12]. We focused on question types that could be automatically scored, and so we did not include fillin-the-blank or open-ended questions. Subsections below provide additional detail about the methodology of our study:

\section{Caption Stimuli at Various Accuracy (WER) Levels}

While we are interested in the design of software for mobile devices (e.g. tablets) to enable DHH users to view captions during one-on-one meetings with hearing colleagues, in order to execute experiments for this study consistently, we generated a set of videos simulating a mock business meeting, to present as stimuli. An actor portrayed a "human resources" officer in a company discussing strategies for hiring more employees; he sat at a desk and addressed the camera (Figure 1), to simulate for the DHH participant the experience of sitting across a table from a hearing colleague. The script was divided into 12 paragraphs, each recorded as a 30 -second video. The reading-complexity of the script was at the $8^{\text {th }}$-grade level (13-14 years old), according to the FleschKincaid Grade Level metric [10] (mean 8.6, SD 2.28).

Captions were superimposed on the video as in Figure 1. In that figure, the speaker was actually saying: "...they want, what their role will be during the internship experience, requirements..." A maximum of three lines of text appear onscreen at a time, following recommendations of [23], and words were appended to the caption stream individually or in short phrases, as in real-time captioning services, e.g. [8].

To produce text captions for the videos at varying levels of accuracy, we began by processing the audio using the Sphinx ASR engine [24], which is an oft-used open source package, but its output is less accurate than the state of the art [16, 43]. We wanted to generate a first draft of each caption text with many errors - so that we could manually correct the text, to produce versions of the caption text at different accuracy levels. As discussed above, ASR accuracy is generally conveyed as Word Error Rate (WER), which is based on the number of substitutions, insertions, and deletions needed to correct words in a transcript when compared to the actual script. Informally, accuracy can be considered the inverse of WER, i.e. $20 \%$ WER corresponding to $80 \%$ accuracy.

We wanted stimuli with three levels of WER (best to worst):

- Human WER: When humans transcribe speech into text, they also make errors, with a typical WER of 5.1\% [37]. Therefore, we set the Human WER level to 5\%. 
- Cloud WER: We considered the performance of modern commercial ASR cloud services when used in a realistic office meeting environment, with conversational style speech, and without near-face microphones. Based on inhouse tests with IBM Watson Speech-To-Text ${ }^{1}$ and Microsoft Bing Speech ${ }^{2}$, we observed approximately 20\% WER for our stimuli. Based on the trend of ASR accuracy improving over time, we wanted to select a slightly better level than current cloud-based ASR engines. Therefore, we set the Cloud WER level to $15 \%$.

- Desktop WER: We selected $\sim 50 \%$ WER as our low condition, which was comparable to accuracy of an ASR for DHH users reported in [30] and to accuracy of modern ASR in settings without good-quality microphones, for multi-speaker conversation, or without speaker training [44, 45]. After running Sphinx ASR on a Desktop system, we used SCLITE [9] to align the ASR transcript to the actual script; the WER of the output was $47.71 \%$.

For all of our 12 stimuli video caption texts, we produced three versions of each (at each WER level): To ensure the errors were realistic and that stimuli at each higher WER level were monotonically worse, we began with the Sphinx ASR output, and we randomly replaced text errors with the correct words, until the desired WER level was achieved. Due to different word-count of each video, we produced versions of each with scores as close as possible to our target WER levels. The mean WER of our modified stimuli videos are: $6.15 \%$ for Human, $15.61 \%$ for Cloud, and $47.71 \%$ for Desktop. With 12 videos produced at 3 WER levels, we had 36 captioned videos to be displayed in the study.

\section{Grouping Participants into Literacy Levels}

To evaluate the literacy skill of participants, we considered the Wechsler Adult Intelligence Scale (WAIS ${ }^{3}$ ) or Michigan Test of English Language Proficiency (MTELP ${ }^{4}$ ) but did not select these due to long test length or use of audio stimuli. Instead, we used the Wide Range Achievement Test 4th Edition (WRAT45), which had been validated with DHH users [39]. The sentence comprehension subscale of WRAT is brief and can be administered without audio. We needed to partition our participants into discrete literacy ranges, but we were unable to find prior research to suggest appropriate thresholds, specifically for the DHH population. WRAT scores (age-adjusted) range from 55 to 145 . Knowing that DHH individuals score lower on this test than the national average (100) [39], we defined three levels for our study:

- WRAT-L: Low scores between 55 and 65

- WRAT-M: Medium scores between 66 and 80

- WRAT-H: High scores of 81 and higher

\footnotetext{
${ }^{1} \mathrm{https} / / /$ www.ibm.com/watson/developercloud/speech-to-text.html

${ }^{2}$ https://www.microsoft.com/cognitive-services/en-us/speech-api

${ }^{3} \mathrm{http}: / / \mathrm{www}$.pearsonclinical.com/psychology/products/100000392/ wechsler-adult-intelligence-scalefourth-edition-wais-iv.html

${ }^{4} \mathrm{http} / / /$ cambridgemichigan.org/institutions/products-services/ tests/placement-progress/mtelp-series/

${ }^{5} \mathrm{http} / / /$ www.pearsonclinical.com/education/products/100001722/ wide-range-achievement-test-4--wrat4.html
}

\section{Data Collection Methodology}

Our participants were recruited at a university through social media, e-mail, and flyers. Our screening criteria included two questions: "Are you Deaf or Hard-of-Hearing?" and "Do you use captions when viewing television?" Participants who answered affirmatively to both met a research assistant (a native ASL signer) to participate in the study in a private office, and they received \$40 USD compensation for the 60minute session. Our 107 participants' ages ranged from 18 to 30 , and they included 48 women. Participants self-identified as Deaf (69), Hard-of-Hearing (36), or Other (2).

After the demographic questionnaire, participants completed the sentence comprehension sub-scale of the WRAT4. Our participants scored lower than the U.S. norm (100), with a mean standardized score of 70.6 ( $\mathrm{SD}=12.89$; range 55-111). Participants were grouped into three levels: 27 in WRAT-L (mean=56.48, SD=1.83), 50 in WRAT-M (mean=67.8, $\mathrm{SD}=3.97)$, and 30 in WRAT-H (mean=87.97, $\mathrm{SD}=8.3)$.

Next, participants viewed the captioned video stimuli, and they responded to questions after each video. Each participant viewed the 12 videos in chronological order, with a Latin-squares schedule used to rotate the three WER conditions. Thus, each participant saw four videos at each WER level. A PHP application on a MacBook Pro 15-inch laptop displayed the videos and collected participants' responses, with participants clicking a "next" button to advance after watching videos or answering questions.

After each video clip, we asked participants four multiplechoice comprehension questions (f-g in our hypothesis list) and a variety of questions asking them to judge the quality of the captions (a-e). Videos and questions are available as supplemental online information on the ACM digital library.

\section{RESULTS AND DISCUSSION}

This section begins with results for $\mathbf{H 1}$ (discriminative ability of probes), with subsections focusing on the results for each question type (probe), corresponding to each sub-hypothesis (a through g). This is followed by a discussion of $\mathrm{H} 1$ results, and then separate results and discussion sections appear for H2 (literacy bias of probes). Table 1 summarizes and organizes the main findings of this paper, and this table may be a sufficient reference for most readers, who may choose to skip ahead to the later "Discussion of H1" section.

Detailed Results for H1: Discriminative Ability of Probes H1 focused on whether each question type could distinguish captions of different quality levels (Human, Cloud, Desktop). We examine this hypothesis separately, for each WRAT literacy level of participants. For this reason, Figures 2-8 in this section contain three faceted subgraphs, one for each WRAT level. For each WRAT literacy subgroup, KruskalWallis tests were performed to determine if there were statistical differences between captions at each accuracy level. Within each subgraph, scores are displayed for each caption accuracy level. Error bars represent standard error, and the statistical significance brackets drawn above each 


\begin{tabular}{|c|c|c|}
\hline Sub-Hypothesis & H1: Discriminative Ability of Probe & H2: Literacy Bias of Probe \\
\hline $\begin{array}{l}\text { a) Noticed Errors in the } \\
\text { Captions (Boolean) }\end{array}$ & $\begin{array}{l}\text { H1-a: Worked somewhat for WRAT-M only. } \\
\text { Useful only for WRAT-M participants (and only } \\
\text { for distinguishing "Human" high-accuracy captions } \\
\text { from the other caption accuracy levels). }\end{array}$ & $\begin{array}{l}\text { H2-a: Supported. There was significant bias: } \\
\text { Participants in each higher WRAT literacy group } \\
\text { reported that they noticed more errors, than did } \\
\text { the participants in each lower literacy group. }\end{array}$ \\
\hline $\begin{array}{l}\text { b) Errors Prevented } \\
\text { Understanding (Likert) }\end{array}$ & $\begin{array}{l}\text { H1-b: Worked well for WRAT-H and somewhat } \\
\text { for WRAT-M: Great for WRAT-H (distinguished } \\
\text { all caption accuracy levels), less useful for WRAT- } \\
\text { M participants (only distinguished "Desktop" low- } \\
\text { accuracy captions from the other accuracy levels). }\end{array}$ & $\begin{array}{l}\text { H2-b: Not Supported. No measurable bias } \\
\text { was observed for responses to this question type } \\
\text { across the different WRAT groups. }\end{array}$ \\
\hline $\begin{array}{l}\text { c) ASR Did a Good Job } \\
\text { (Likert) }\end{array}$ & $\begin{array}{l}\text { H1-c: Worked somewhat for WRAT-H and } \\
\text { WRAT-M. For WRAT-H and WRAT-M } \\
\text { participants, it only distinguished the "Desktop" } \\
\text { low-accuracy captions from the other levels. }\end{array}$ & $\begin{array}{l}\text { H2-c: Partially Supported. Significant bias } \\
\text { towards more critical scores was found only in } \\
\text { WRAT-H participants, but there was no } \\
\text { significant difference between WRAT-M and -L. }\end{array}$ \\
\hline $\begin{array}{l}\text { d) Evaluation of Accuracy } \\
\text { (Ordinal Scale) }\end{array}$ & $\begin{array}{l}\text { H1-d: Worked well for WRAT-H, somewhat for } \\
\text { WRAT-M, and barely for WRAT-L. Great for } \\
\text { WRAT-H participants (distinguished all caption } \\
\text { accuracy levels). Less useful for WRAT-M } \\
\text { (distinguished "Desktop" captions from others) or } \\
\text { WRAT-L (distinguished Human vs. Desktop). }\end{array}$ & $\begin{array}{l}\text { H2-d: Partially Supported. Significant bias } \\
\text { towards more critical scores was found only in } \\
\text { WRAT-H participants, but there was no } \\
\text { significant difference between the scores from } \\
\text { WRAT-M and WRAT-L participants. }\end{array}$ \\
\hline $\begin{array}{l}\text { e) Evaluation of Accuracy } \\
\text { (Numeric) }\end{array}$ & $\begin{array}{l}\text { H1-e: Worked well for WRAT-M and somewhat } \\
\text { for WRAT-H. Great for WRAT-M (distinguished } \\
\text { all caption accuracy levels). Less useful for } \\
\text { WRAT-H participants (distinguished "Desktop" } \\
\text { low-accuracy captions from the other levels). }\end{array}$ & $\begin{array}{l}\text { H2-e: Not Supported. No measurable bias was } \\
\text { observed for responses to this question type } \\
\text { across the different WRAT groups. }\end{array}$ \\
\hline $\begin{array}{l}\text { f) Comprehension Quiz } \\
\text { Success (Multiple-Choice) }\end{array}$ & $\begin{array}{l}\text { H1-f: Worked somewhat for WRAT-M and } \\
\text { WRAT-L and barely for WRAT-H. Useful for } \\
\text { WRAT-M and WRAT-L participants } \\
\text { (distinguished "Desktop" low-accuracy captions } \\
\text { from other levels). Less useful for WRAT-H } \\
\text { (distinguished Human vs. Desktop only). }\end{array}$ & $\begin{array}{l}\text { H2-f: Supported. There was significant bias: } \\
\text { Participants in each higher WRAT literacy group } \\
\text { had higher comprehension question success } \\
\text { scores than did participants in each of the lower } \\
\text { WRAT groups. }\end{array}$ \\
\hline $\begin{array}{l}\text { g) Comprehension Quiz } \\
\text { Time (Seconds) }\end{array}$ & $\begin{array}{l}\text { H1-g: This question did not work well. Not } \\
\text { effective at discriminating between any caption } \\
\text { accuracy levels, for any WRAT group. }\end{array}$ & $\begin{array}{l}\text { H2-g: Not Supported. No measurable bias was } \\
\text { observed for responses to this question type } \\
\text { across the different WRAT groups. }\end{array}$ \\
\hline
\end{tabular}

Table 1. Summary of results for $\mathrm{H} 1$ and $\mathrm{H} 2$, for each sub-hypothesis corresponding to the seven question types (a-g).

graph indicate the result of Mann-Whitney U-tests (also known as Wilcoxon rank-sum tests) for post-hoc pairwise statistical significance testing with Bonferroni corrections, with p-values indicated as “***” (0 to 0.001$)$, “**” $(<0.01)$, "*” $(<0.05)$, or "NS." not significant. When the reader sees statistical differences between the bars in a subgraph, this suggests that $\mathrm{H} 1$ was supported for that question type, for data collected from participants at that WRAT level.

\section{H1-a: Noticed Errors in the Captions (Boolean)}

Figure 2 displays mean scores after the following conversion: $\mathrm{No}=0$, Yes $=1$. Results from statistical tests are as follows:

- WRAT-L: No statistical difference observed ( $p>0.05)$

- WRAT-M: In the case of participants with medium literacy levels, there were significant differences between captions of different accuracy levels $\left(\chi^{2}(2)=11.211, p=0.003678\right)$, and pairwise tests revealed one statistical difference: Desktop vs. Human.

- WRAT-H: No statistical difference observed ( $\mathrm{p}>0.05)$.

H1-b: Errors Prevented Understanding (Likert)

Figure 3 (next page) displays the percentage of respondents who selected each option (e.g. strongly agree) in response to this question. Results from Kruskal-Wallis and post hoc tests (after standard conversion of Likert levels to integers, e.g. strongly disagree $=1$, disagree $=2$, and so on) were as follows:

- WRAT-L: No statistical difference observed ( $\mathrm{p}>0.05$ )

- WRAT-M: There were significant differences between captions of different accuracy levels $\left(\chi^{2}(2)=18.894\right.$, $p=7.894 e-05)$, and pairwise tests revealed differences for: Desktop vs. Cloud and Desktop vs. Human.

- WRAT-H: For participants with high literacy levels, there were significant differences between captions of different accuracy levels $\left(\chi^{2}(2)=42.635, p=5.521 e-10\right)$, with significant differences between all pairs, post-hoc.

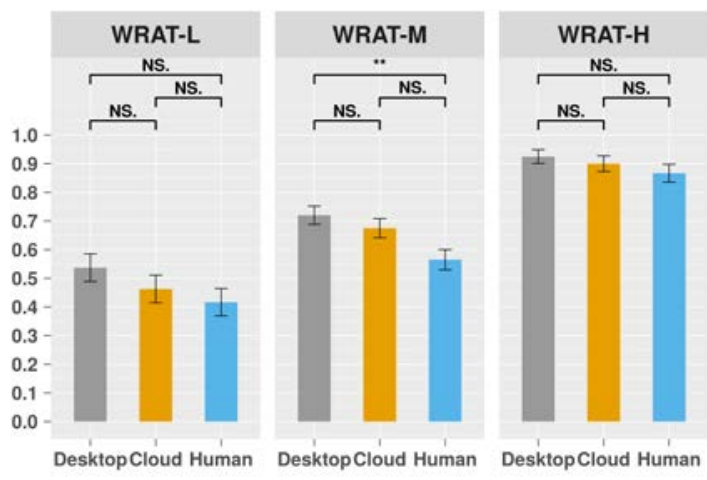

Figure 2. H1-a for Noticed Errors (Boolean) 


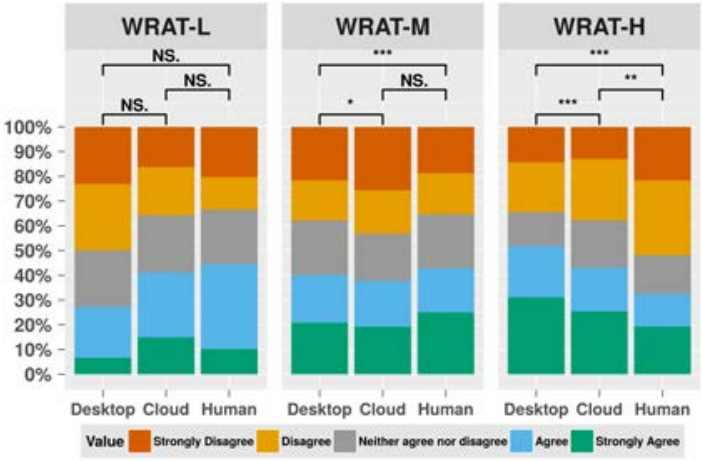

Figure 3. H1-b for Errors Prevented Understanding (Likert)

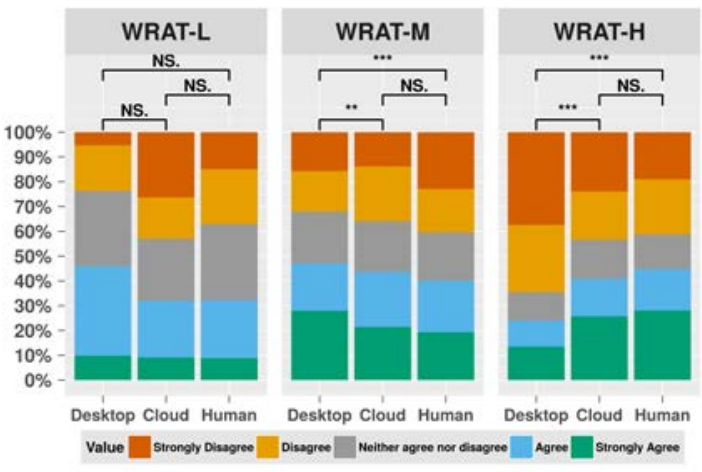

Figure 4. H1-c for ASR Did a Good Job (Likert)

H1-c: ASR Did a Good Job (Likert)

Figure 4 displays the percentage of respondents who selected each option. Results from Kruskal-Wallis and post hoc tests (converting levels to integers as in $\mathrm{H} 1-\mathrm{b}$ ) were as follows:

- WRAT-L: No statistical difference observed $(\mathrm{p}>0.05)$.

- WRAT-M: There were significant differences between captions of different accuracy levels $\left(\chi^{2}(2)=29.005\right.$, $p=5.032 e-07)$, and pairwise tests revealed differences for: Desktop vs. Cloud and Desktop vs. Human.

- WRAT-H: There were similar results for participants with high literacy levels $\left(\chi^{2}(2)=60.897, p=5.975 e-14\right)$.

H1-d: Evaluation of Accuracy (Ordinal Scale)

Figure 5 displays the percentage who selected each choice in response to "The caption accuracy was:" After executing the Kruskal-Wallis and post hoc tests (after conversion of scalar levels to integers, e.g. "Zero" $=1$, "Low" $=2$, etc.) we found:

- WRAT-L: There were significant differences between captions of different accuracy levels $\left(\chi^{2}(2)=7.0291\right.$, $p=0.02976$ ), with a difference for Desktop vs. Human.

- WRAT-M: There were significant differences between captions of different accuracy levels $\left(\chi^{2}(2)=39.118\right.$, $p=3.203 e-09$ ), and pairwise tests revealed differences: Desktop vs. Cloud and Desktop vs. Human.

- WRAT-H: There were significant differences between captions of different accuracy levels $\left(\chi^{2}(2)=66.888\right.$, $p=2.988 e-15)$, with all pairwise differences significant.

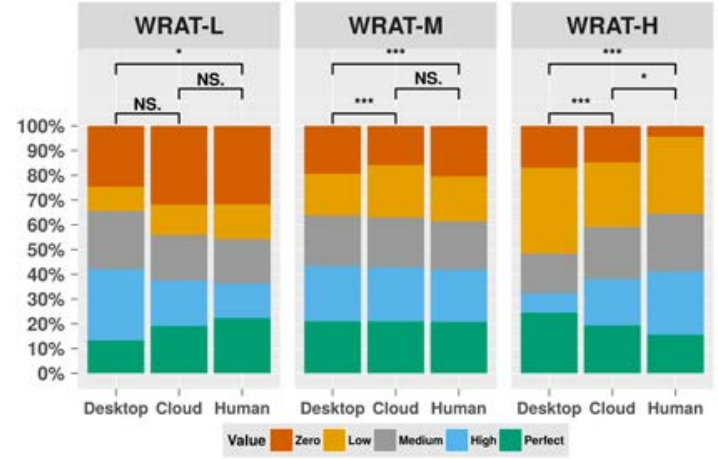

Figure 5. H1-d for Evaluation of Accuracy (Ordinal Scale)

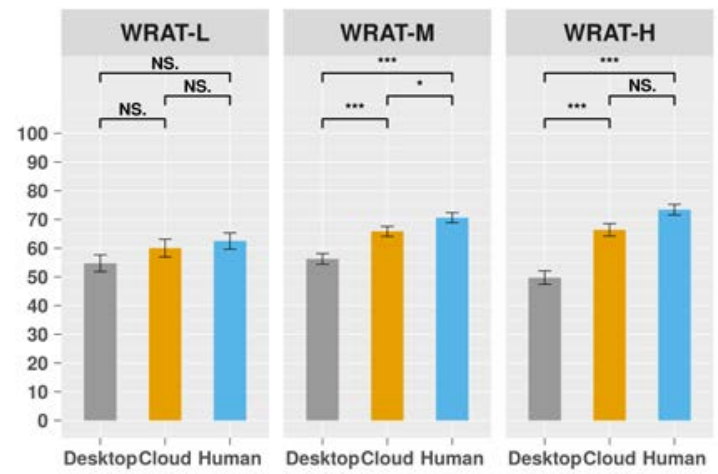

Figure 6. H1-e for Evaluation of Accuracy (Numeric)

H1-e: Evaluation of Accuracy (Numeric)

Figure 6 displays the mean scores when participants guessed a numeric percentage value for each caption's accuracy. Results from statistical tests were as follows:

- WRAT-L: No statistical difference observed $(\mathrm{p}>0.05)$.

- WRAT-M: There were significant differences between captions of different accuracy levels $\left(\chi^{2}(2)=38.84\right.$, $p=3.681 e-09)$, with all pairwise differences significant.

- WRAT-H: There were significant differences between captions of different accuracy levels $\left(\chi^{2}(2)=55.886\right.$, $p=7.32 e-13)$, with significant differences only for: Desktop vs. Cloud and Desktop vs. Human.

\section{H1-f: Comprehension Quiz Success (Multiple-Choice)}

Figure 7 (next page) displays the overall percentage of correct answers. Results from statistical tests were as follows:

- WRAT-L: There were significant differences between different accuracy levels $\left(\chi^{2}(2)=11.554, p=0.003097\right)$, with significant pairwise differences only for: Desktop vs. Cloud and Desktop vs. Human.

- WRAT-M: There were significant differences between captions of different accuracy levels $\left(\chi^{2}(2)=27.481\right.$, $p=1.078 e-06$ ), with significant pairwise differences only for: Desktop vs. Cloud and Desktop vs. Human.

- WRAT-H: There were significant differences between different accuracy levels $\left(\chi^{2}(2)=14.519, p=0.0007033\right)$, with a pairwise difference between Desktop vs. Human. 


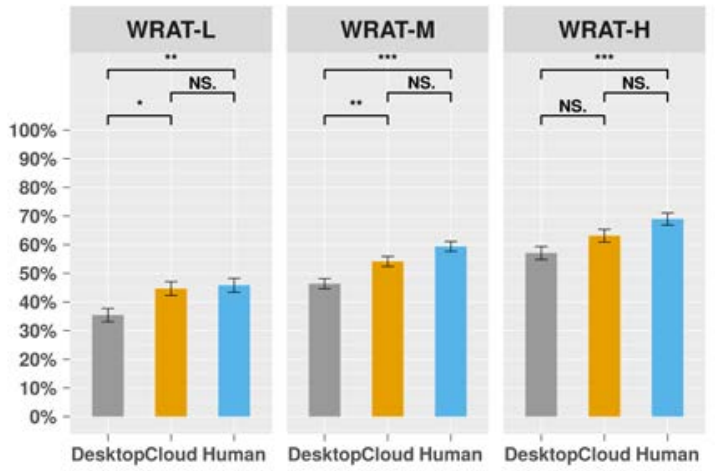

Figure 7. H1-f for Comprehension Quiz Success (Multiple Choice)

\section{H1-g: Comprehension Quiz Time (Seconds)}

Figure 8 shows the time in seconds for participants to answer four comprehension questions for each video. There were no significant differences $(\mathrm{p}>0.05)$ for any WRAT group.

\section{Discussion of H1: Discriminative Ability of Probes}

The reader may find it helpful to consult the summary of results presented earlier in Table 1. The most successful probe (with significance across all WER levels for WRAT-H and across some levels for WRAT-M and WRAT-L) asked participants to evaluate caption accuracy on an ordinal scale (H1-d). Six of the seven question types were effective, to some degree, at discriminating caption accuracy levels, when used with participants in the WRAT-H high literacy group (with best results for H1-b and H1-d), and to a lesser degree with WRAT-M participants (best results for H1-e).

A major trend in the results for $\mathrm{H} 1$ was that few question types were effective for use with participants with low literacy (WRAT-L). For these users, comprehension question success (H1-f) distinguished low accuracy captions from others, and estimating caption accuracy on an ordinal scale (H1-d) was only able to distinguish large accuracy differences (i.e. Desktop vs. Human). In the case of subjective question types (a-e), WRAT-L participants may have already been struggling to read captions containing errors, and on top of this, many of these question types require "meta-literacy," i.e. readers had to introspect as to whether their difficulty in understanding the caption was due to imperfections in the caption itself or due to their limited literacy skills. For comprehension questions (f-g), we suspect that the complexity of the questions asked or of the original caption text may have been poorly targeted to the literacy skill level of participants in the WRAT-L group.

We also noted that our participants generally struggled to discriminate between the Cloud and Human levels of WER, e.g. for probes (a, b, c, f, and g). Since the WER for Human $(5 \%)$ and Cloud $(15 \%)$ were more similar than for Desktop $(50 \%)$, it may have been more difficult to distinguish them.

\section{Detailed Results for H2: Literacy Bias of the Probe}

$\mathrm{H} 2$ focused on whether participants at different literacy levels would have significant differences in their response scores, which could indicate a "literacy bias for each probe," such

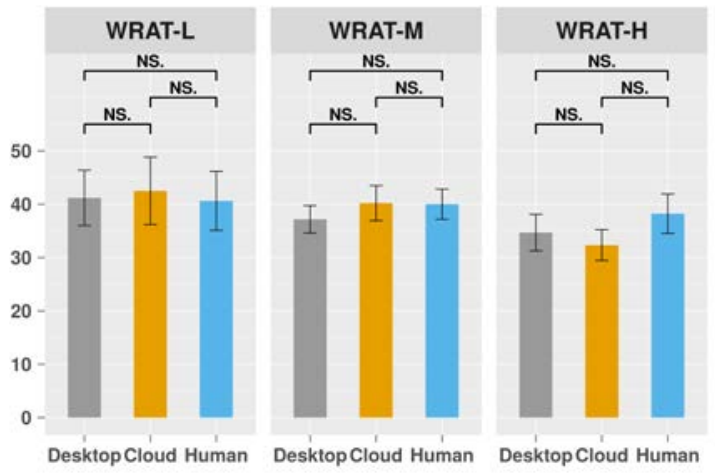

Figure 8. H1-g for Comprehension Quiz Time (Seconds)

that participants with higher literacy levels might be more critical judges, in keeping with prior research on ASL in [18].

Detailed results for $\mathrm{H} 2$ appear below: Some readers may instead wish to consult the summary of results in Table 1 and skip ahead to the "Discussion of H2" section below.

Figures 9-14 in this section each consist of a simple bar graph of response scores (across all stimuli in the study) for the three WRAT groups. All Boolean or scalar response scores are converted to integers, following conversions explained above for H1-a to H1e. The three groups were compared using a Kruskal-Wallis test, followed by post-hoc pairwise Mann-Whitney U-tests with Bonferroni corrections. The brackets displayed atop each graph show the result of these pairwise comparisons, with p-values indicated as "***" $(0$ to $0.001)$, “**” $(<0.01)$, “*” $(<0.05)$, or "NS.” not significant.

H2-a: Noticed Errors in the Captions (Boolean)

Figure 9 (next page) displays the mean scores for each WRAT level. The Kruskal-Wallis test indicated significance: $\left[\chi^{2}(2)=143.17, p<2.2 e-16\right]$. The mean and standard errors are: WRAT-L: $\mu=0.47, \mathrm{SE}=0.03$; WRATM: $\mu=0.65, \mathrm{SE}=0.02$; and WRAT-H: $\mu=0.9, \mathrm{SE}=0.02$. H2-a is supported: Participants in each literacy group noticed more errors than did participants in lower literacy groups. In fact, we found that WRAT-H participants reported noticing errors in captions at almost twice the rate of the WRAT-L participants in our study.

\section{H2-b: Errors Prevented Understanding (Likert)}

Figure 10 (next page) displays the median scores for each WRAT level as a boxplot. The red dots represent outliers in the data. The Kruskal-Wallis test did not indicate significance: $\left[\chi^{2}(2)=0.1598, p=0.9232\right]$, with a median score of 3 (Neither agree nor disagree) for all WRAT levels. WRAT-H and WRAT-M had a mode of 3 , while WRAT-L had a mode of 4 (Agree). H2-b is not supported.

H2-c: ASR Did a Good Job (Likert)

Figure 11 (next page) displays the median scores for each WRAT level as a boxplot. The Kruskal-Wallis test indicated significance: $\left[\chi^{2}(2)=15.091, p=0.0005286\right]$, with a median score of 3 (Neither agree nor disagree) and mode of 4 (Agree) for all WRAT levels. H2-c is supported: WRAT-H participants had lower scores than WRAT-M or WRAT-L. 


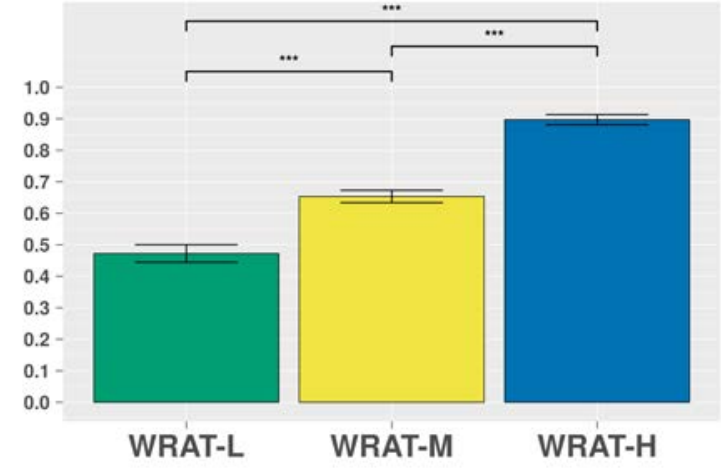

Figure 9. H2-a for Noticed Errors in Captions (Boolean)

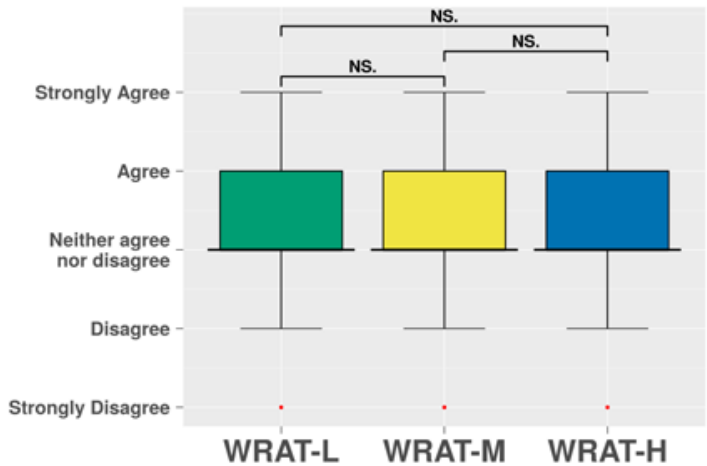

Figure 10. H2-b for Errors Prevented Understanding (Likert)

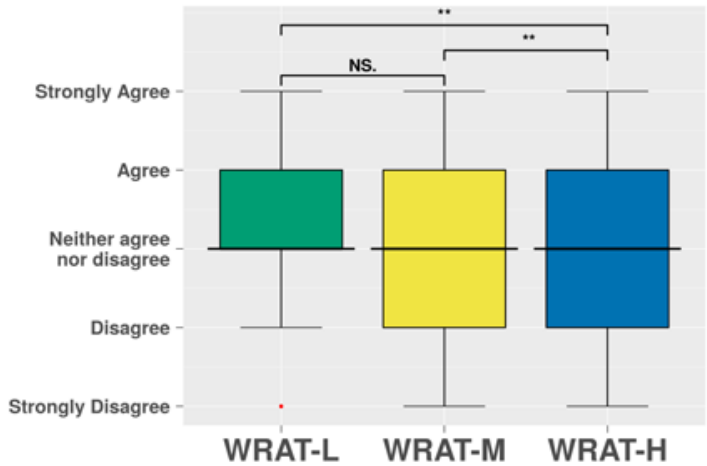

Figure 11. H2-c for ASR Did a Good Job (Likert)

H2-d: Evaluation of Accuracy (Ordinal Scale)

Figure 12 displays the median scores for each WRAT level as a boxplot. The Kruskal-Wallis test indicated significance: $\left[\chi^{2}(2)=7.539, p=0.02306\right]$, with a median and mode of 3 (Medium) for all WRAT levels. H2-d is supported: WRATH participants had lower scores than WRAT-M or WRAT-L.

H2-e: Evaluation of Accuracy (Numeric)

Figure 13 displays the mean scores for each WRAT level. The Kruskal-Wallis test did not indicate significance: $\left[\chi^{2}(2)=2.9637, p=0.2272\right]$, with means and standard errors: WRAT-L: $\mu=59.1, \mathrm{SE}=1.71$; WRAT-M: $\mu=64.24, \mathrm{SE}=1.05$; and WRAT-H: $\mu=63.19, \mathrm{SE}=1.05$. H2-e is not supported.

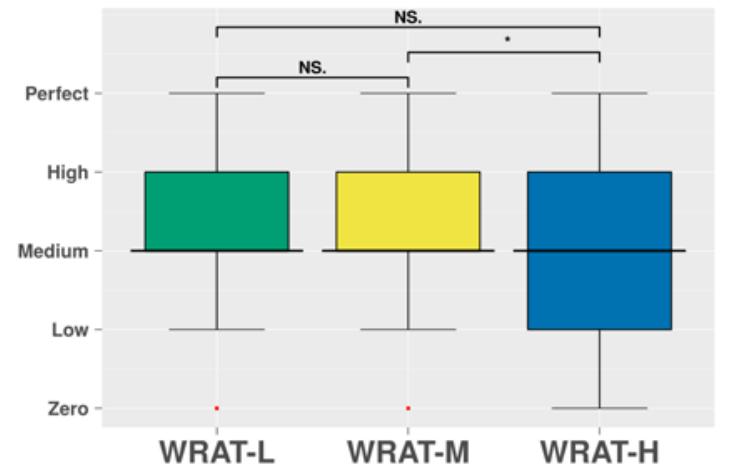

Figure 12. H2-d for Accuracy of Evaluation (Ordinal Scale)

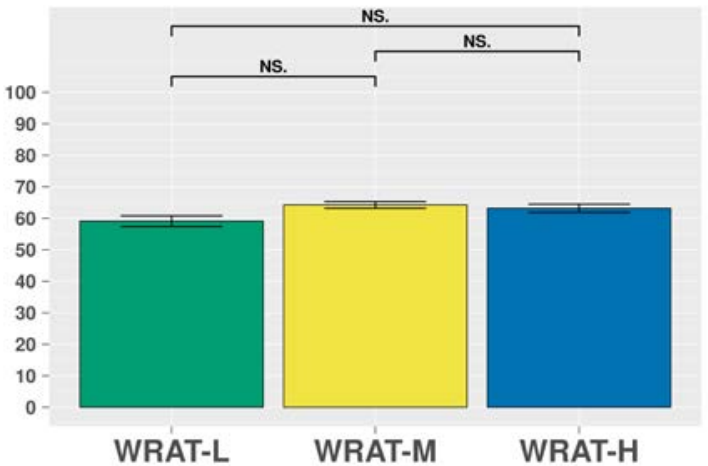

Figure 13. H2-e for Evaluation of Accuracy (Numeric)

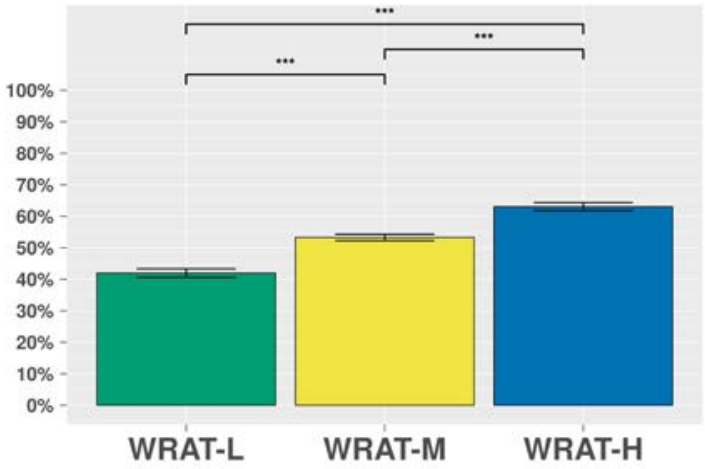

Figure 14. H2-f for Comprehension Quiz Success (Mult. Choice)

H2-f: Comprehension Quiz Success (Multiple-Choice)

Figure 14 displays the mean for each WRAT level. The Kruskal-Wallis test indicated significance: $\left[\chi^{2}(2)=121.74\right.$, $p<2.2 e-16]$, with mean and standard errors: WRAT-L: $\mu=0.42, \mathrm{SE}=0.01$; WRAT-M: $\mu=0.53, \mathrm{SE}=0.01$; and WRAT$\mathrm{H}: \mu=0.63, \mathrm{SE}=0.01$. H2-f is supported: Participants had higher comprehension question success scores than those participants in lower WRAT literacy groups.

H2-g: Comprehension Quiz Time (Seconds)

Since the analysis for $\mathbf{H 1}$ indicated no significance at all, there is no need to display another figure (Figure 8). The Kruskal-Wallis test did not indicate significance: $\left[\chi^{2}(2)=1.6128, p=0.4465\right]$. H2-g is not supported. 


\section{Discussion of H2: Literacy Bias of the Probes}

Again, the reader may find it helpful to consult the summary of results presented earlier in Table 1. H2 was focused on whether there was a "bias" such that participants at different literacy skill levels had overall higher or lower scores, for each question type. Question types with this literacy bias are not necessarily bad questions to use in evaluation studies of captioning with DHH users, but researchers should report participants' literacy levels and consider this bias when comparing results across studies.

Results for $\mathrm{H} 2$ varied across our different question types: Two probes (a and $\mathrm{f}$ ) had significant differences across participants at all three WRAT levels, and two (c and d) had significant differences only for WRAT-H participants. Of these, participants with higher literacy gave more negative subjective responses: They were more likely to report noticing errors in captions ( $\mathrm{H} 2-\mathrm{a})$, did not agree that ASR did a good job (H2-c), and had lower estimates of caption accuracy on ordinal scales (H2-d). Finally, as expected, participants with higher literacy had higher scores on the comprehension quiz success $(\mathrm{H} 2-\mathrm{f})$.

We did not observe significant bias for: H2-b errors prevented understanding (Likert item), H2-e evaluation of accuracy on a numeric scale, nor H2-g comprehension quiz time. In the case of $\mathrm{H} 2-\mathrm{b}$, we speculate that the question related to two factors: whether there had been many errors in the captions and the user's opinion as to whether this affected their understanding - perhaps in the case of high literacy users, they did not believe they were affected by the errors. For $\mathrm{H} 2-\mathrm{e}$, it was notable that while significant bias was measured for the ordinal-scale version of this question (H2-d), this trend was not observed for the numeric version; we speculate that the process of numeric estimation was less prone to this bias effect. Finally, although no bias effect was observed for comprehension quiz time, this had been a poor indicator of caption accuracy in H1-g (Figure 8).

\section{CONCLUSIONS, LIMITATIONS, AND FUTURE WORK}

Through a lab study with 107 DHH participants evaluating ASR-based captions of various known accuracy levels, we have compared the scores recorded from various question types. We found that some question types are effective at distinguishing accuracy levels of captions, for responses collected from participants within particular literacy ranges. We also found that for some question types, participants with higher literacy levels give more critical subjective responses and have higher comprehension question scores. Based on this, we provide the following methodological recommendations to researchers conducting evaluations of captioning technology in studies with DHH participants:

- Because the literacy level of participants influences their response scores, researchers should report the literacy level of the participants in their studies - or control for this factor through reported screening criteria.

- If participants' literacy levels are diverse, researchers should report results separately for those at each level.
- When selecting questions to include in the study, researchers should consider the literacy level of their participants and the accuracy level of their caption stimuli, to select an appropriate question type capable of discriminating caption quality (i.e. consult Table 1).

- Given that question types may differ in their ability to distinguish caption quality at different accuracy levels, researchers should consider their caption accuracy, e.g. using metrics such as WER, and report these values.

- Since comprehension question scores are based on several factors, including: the quality of the original text, the difficulty of the question, and the reading skill level of the respondent - such questions must be appropriately tailored to the participants' literacy skills. For instance, participants with above-average fluency may better infer the erroneous/missing information in captions.

There were several limitations to our study: We chose the ubiquitous WER as an accuracy metric, but newer metrics that weigh the importance of each word may better represent accuracy perceptions of DHH users [19]. Our caption stimuli consisted of a single genre (simulation of a meeting) with a script at a particular reading level (as measured by Flesch-Kincaid's formulas); however, we did not experiment with genres or reading-levels of stimuli in this study, and doing so might reveal unforeseen effects. Further, while we used WRAT to group participants into literacy levels, with our lowest group including the lowest range of WRAT scores, we could have recruited greater numbers of participants with high literacy levels, to enable more subdivision at the higher end of the WRAT scale - or utilized a different literacy test with greater ability to distinguish individuals at the extremes of the literacy scale. Our study included participants recruited from a university campus; researchers should use caution when generalizing our results to other DHH users, e.g. younger students or older adults in the workforce. Finally, our study did not include all possible question-type probes, e.g. open-ended questions or eye-tracking metrics, as in some prior work.

This methodological study has been conducted as part of a larger research project at our lab to investigate the use of ASR-based captioning for use by DHH individuals in oneon-one meetings with hearing colleagues, e.g. in workplace settings. In future work, we plan to design and develop a prototype system, which we will evaluate in user studies. We will make use of the methodological guidelines above, and we may generate revisions to them based on our experiences in conducting further studies with DHH users.

\section{ACKNOWLEDGEMENTS}

This material is based upon work supported by the National Science Foundation Graduate Research Fellowship under Grant No. 1746056, and by a Google Faculty Research Award and by the National Technical Institute for the Deaf. We are grateful for the contribution of Christopher Caulfield in designing stimuli, and for Kasmira Patel and Amnolvir Kaur in the collection of data from participants. 


\section{REFERENCES}

1. John Albertini, Connie Mayer. 2011. Using miscue analysis to assess comprehension in deaf college readers. J Deaf Stud Deaf Edu 16, 1 (2011), 35. http://dx.doi.org/10.1093/deafed/enq017

2. Larwan Berke, Christopher Caulfield, Matt Huenerfauth. 2017. Deaf and hard-of-hearing perspectives on imperfect automatic speech recognition for captioning one-on-one meetings. In Proceedings of the 19th International ACM SIGACCESS Conference on Computers and Accessibility (ASSETS '17). ACM, NY, NY, USA. https://doi.org/10.1145/3132525.3132541

3. Frank Bowe. 2002. Deaf and hard of hearing Americans' instant messaging and e-mail use: A national survey. American Annals of the Deaf 147, 4 (2002), 6-10. http://dx.doi.org/10.1353/aad.2012.0251

4. Michael Stinson, Lisa Elliot, Ronald Kelly. 2017. Deaf and hard-of-hearing high school and college students' perceptions of speech-to-text and interpreting/note taking services and motivation. J Dev Phys Disabil, 29, 1, 131-152. https://doi.org/10.1007/s10882-017-9534-4

5. Ana-Belen Dominguez, Jesus Alegria. 2010. Reading mechanisms in orally educated deaf adults. Journal of deaf studies and deaf education, 15(2): 136-148. https://doi.org/10.1093/deafed/enp033

6. Anita Cremers, Marlies Welbie, Kim Kranenborg, Harriët Wittink. 2017. Deriving guidelines for designing interactive questionnaires for low-literate persons. Universal Access in the Information Society 16, 1, 161172. http://dx.doi.org/10.1007/s10209-015-0431-2

7. Lisa Elliot, Michael Stinson, James Mallory, Donna Easton, Matt Huenerfauth. 2016. Deaf and hard of hearing individuals' perceptions of communication with hearing colleagues in small groups. In Proceedings of the 18th International ACM SIGACCESS Conference on Computers and Accessibility (ASSETS '16). ACM, NY, 271-272. http://dx.doi.org/10.1145/2982142.2982198

8. Lisa Elliot, Michael Stinson, Barbara McKee, Victoria Everhart, Pamela Francis. 2001. College students' perceptions of the C-Print speech-to-text transcription system. Journal of deaf studies and deaf education 6, 4, 285-298. http://dx.doi.org/10.1093/deafed/6.4.285

9. Jon Fiscus. 2007. Speech recognition scoring toolkit (SCTK). Retrieved July 1, 2017 from http://www.itl.nist.gov/iad/mig/tools/

10. Rudolph Flesch. 1948. A new readability yardstick. Journal of applied psychology 32, 3 (1948), 221-233.

11. Yashesh Gaur, Florian Metze, Yajie Miao, Jeffrey Bigham. 2015. Using keyword spotting to help humans correct captioning faster. In Conf. of Int'l Speech Communication Assoc. (Interspeech'15). 2829-2833.

12. Stephen Gulliver, George Ghinea. 2003. How level and type of deafness affect user perception of multimedia video. Universal Access in the Information Society 2, 4, 374-386. http://dx.doi.org/10.1109/ICME.2002.1035891

13. Rebecca Perkins Harrington, Gregg Vanderheiden. 2013. Crowd caption correction (ccc). In Proc. Conf. on
Computers and Accessibility (ASSETS '13). ACM, NY, NY, USA, http://dx.doi.org/10.1145/2513383.2513413

14. Xiodong He, Li Deng, Alex Acero. 2011. Why word error rate is not a good metric for speech recognizer training for the speech translation task? In 2011 IEEE International Conference on Acoustics, Speech and Signal Processing (ICASSP). 5632-5635. http://dx.doi.org/10.1109/ICASSP.2011.5947637

15. Ippei Hisaki, Hiroaki Nanjo, Takehiko Yoshimi. 2010. Evaluation of speech balloon captions for auditory information support in small meetings. In Proceedings of 20th International Congress on Acoustics. 3775-3779.

16. Xuedong Huang, James Baker, Raj Reddy. 2014. A historical perspective of speech recognition. Commun. ACM 57, 1, 94-103. http://dx.doi.org/10.1145/2500887

17. Matt Huenerfauth, Lijun Feng, Noémie Elhadad. 2009. Comparing evaluation techniques for text readability software for adults with intellectual disabilities. In Proceedings of the 11th International ACM SIGACCESS Conference on Computers and Accessibility (Assets '09). ACM, New York, NY, USA, 3-10. http://dx.doi.org/10.1145/1639642.1639646

18. Hernisa Kacorri, Matt Huenerfauth, Sarah Ebling, Kasmira Patel, Kellie Menzies, Mackenzie Willard. 2017. Regression analysis of demographic and technologyexperience factors influencing acceptance of sign language animation. ACM Trans. Access. Comput. 10, 1, Article 3 (April 2017), 33 pages. http://dx.doi.org/10.1145/3046787

19. Sushant Kafle, Matt Huenerfauth. 2016. Effect of speech recognition errors on text understandability for people who are deaf or hard of hearing. In $7 t h$ Workshop on Speech and Language Processing for Assistive Technologies (SLPAT). 20-25. http://scholarworks.rit.edu/other/891/

20. Saba Kawas, George Karalis, Tzu Wen, Richard Ladner. 2016. Improving real-time captioning experiences for deaf and hard of hearing students. In Conf. on Computers and Accessibility (ASSETS '16). ACM, NY, USA, 15-23. http://dx.doi.org/10.1145/2982142.2982164

21. Richard Kheir, Thomas Way. 2007. Inclusion of deaf students in computer science classes using real-time speech transcription. In Proc. of the 12th Annual SIGCSE Conf. on Innovation and Technology in Computer Science Education (ITiCSE '07). ACM, N, NY, USA, 261-265. http://dx.doi.org/10.1145/1268784.1268860

22. Raja Kushalnagar, Walter Lasecki, Jeffrey Bigham. 2012. A readability evaluation of real-time crowd captions in the classroom. In Proceedings of the 14th International ACM SIGACCESS Conference on Computers and Accessibility (ASSETS '12). ACM, NY, NY, USA, 71-78. http://dx.doi.org/10.1145/2384916.2384930

23. Raja Kushalnagar, Walter Lasecki, Jeffrey Bigham. 2014. Accessibility evaluation of classroom captions. $A C M$ Trans. Access. Comput. 5, 3, Article 7 (Jan. 2014), 24 pp. http://dx.doi.org/10.1145/2543578 
24. Paul Lamere, Philip Kwok, Evandro Gouvea, Bhiksha Raj, Rita Singh, William Walker, Manfred Warmuth, Peter Wolf. 2003. The CMU SPHINX-4 speech recognition system. In Conf. on Acoustics, Speech and Signal Processing (ICASSP 2003), Vol. 1., 2-5.

25. Seongjae Lee, Sunmee Kang, Hanseok Ko, Minseok Keum. 2013. Dialogue enabling speech-to-text user assistive agent with auditory perceptual beamforming for hearing-impaired. In Proc. of the IEEE International Conference on Consumer Electronics (ICCE). 360-361. http://dx.doi.org/10.1109/ICCE.2013.6486929

26. Michella Maiorana-Basas, Claudia Pagliaro. 2014. Technology use among adults who are deaf and hard of hearing: a national survey. The Journal of Deaf Studies and Deaf Education 19, 3 (2014), 400-410. http://dx.doi.org/10.1093/deafed/enu005

27. Marc Marschark, John Albertini, Harry Lang. 2002. Educating deaf students: from research to practice (1 ed.). Oxford University Press.

28. Marc Marschark, Greg Leigh, Patricia Sapere, Denis Burnham, Carol Convertino, Michael Stinson, Harry Knoors, Mathijs PJ Vervloed, William Noble. 2006. Benefits of sign language interpreting and text alternatives for deaf students' classroom learning. Journal of deaf studies and deaf education 11, 4 (2006), 421-437. https://doi.org/10.1093/deafed/en1013

29. Tara Matthews, Scott Carter, Carol Pai, Janette Fong, Jennifer Mankoff. 2006. Scribe4Me: Evaluating a mobile sound transcription tool for the deaf. In Proceedings of the Conference on Ubiquitous Computing. Springer, Berlin, 159-176. http://dx.doi.org/10.1007/11853565_10

30. Mohammad Reza Mirzaei, Seyed Ghorshi, Mohammad Mortazavi. 2012. Using augmented reality and automatic speech recognition techniques to help deaf and hard of hearing people. In Proceedings of the 2012 virtual reality international conference. ACM, NY, USA, Article 5, 4 pages. http://dx.doi.org/10.1109/SVR.2012.10

31. Cosmin Munteanu, Heather Molyneaux, Julie Maitland, Daniel McDonald, Rock Leung, Joanna Lumsden, Hélène Fournier. 2012. Tale of two studies: Challenges in field research with low-literacy adult learners in a developed country. In CHI '12 Extended Abstracts on Human Factors in Computing Systems. ACM, NY, NY, USA, 489-504. http://dx.doi.org/10.1145/2212776.2212825

32. Mark Neerincx, Anita Cremers, Judith Kessens, David van Leeuwen, Khiet P. Truong. 2009. Attuning speechenabled interfaces to user and context for inclusive design: technology, methodology and practice. Universal Access in the Information Society (UAIS) 8, 2 (2009), 109-122. http://dx.doi.org/10.1007/s10209-008-0136-X

33. Susan Parault, Heather Williams. 2010. Reading Motivation, Reading Amount, and Text Comprehension in Deaf and Hearing Adults. J Deaf Stud Deaf Edu 15, 2, 120-135. http://dx.doi.org/10.1093/deafed/enp031

34. Soraia Silva Prietch, Napoliana Silva de Souza, Lucia Villela Leite Filgueiras. 2015. Application requirements for deaf students to use in inclusive classrooms. In Proc. of the Latin American Conf. on Human Computer Interaction (CLIHC '15). ACM, NY, NY, USA, Article 5, 8 pages. http://dx.doi.org/10.1145/2824893.2824898

35. Luz Rello, Ricardo Baeza-Yates. 2017. How to present more readable text for people with dyslexia. Univ Access in the Information Society 16, 1 (2017), 2949. http://dx.doi.org/10.1007/s10209-015-0438-8

36. Horacio Saggion, Sanja Štajner, Stefan Bott, Simon Mille, Luz Rello, Biljana Drndarevic. 2015. Making it simplext: Implementation and evaluation of a text simplification system for Spanish. ACM Trans. Access. Comput. 6, 4, Article 14 (May 2015), 36 pages. http://dx.doi.org/10.1145/2738046

37. George Saon, Gakuto Kurata, Tom Sercu, Kartik Audhkhasi, Samuel Thomas, Dimitrios Dimitriadis, Xiaodong Cui, Bhuvana Ramabhadran, Michael Picheny, Lynn-Li Lim, Bergul Roomi, Phil Hall. 2017. English conversational telephone speech recognition by humans and machines. CoRR, abs/1703.02136. http://arxiv.org/abs/1703.02136

38. Brent Shiver, Rosalee Wolfe. 2015. Evaluating alternatives for better deaf accessibility to selected webbased multimedia. In Proc. of the $17^{\text {th }}$ International ACM SIGACCESS Conference on Computers and Accessibility (ASSETS '15). ACM, NY, NY, USA, 223-230. http://dx.doi.org/10.1145/2700648.2809843

39. LeAdelle Phelps, Barbara Branyan. 1990. Academic achievement and nonverbal intelligence in public school hearing-impaired children. Psychol Schs, 27, 210-217.

40. Michael Stinson, Lisa Elliot, Ronald Kelly, Yufang Liu. 2009. Deaf and hard-of-hearing students' memory of lectures with speech-to-text and interpreting/note taking services. The Journal of Special Education 43, 1 (2009), 52-64. http://dx.doi.org/10.1177/0022466907313453

41. Dorothy Jackson, Peter Paul, Jonathan C. Smith. 1997. Prior knowledge and reading comprehension ability of deaf adolescents. J Deaf Stud Deaf Edu, 2, 3, 172-84.

42. Mike Wald. 2005. Using automatic speech recognition to enhance education for all students: Turning a vision into reality. In Proc. of Frontiers in Education Conference. S3G-22-25. http://dx.doi.org/10.1109/FIE.2005.1612286

43. Wayne Xiong, Jasha Droppo, Xuedong Huang, Frank Seide, Mike Seltzer, Andreas Stolcke, Dong Yu, Geoffrey Zweig. 2016. The Microsoft 2016 conversational speech recognition system. CoRR, abs/1609.03528. http://arxiv.org/abs/1609.03528

44. Walter Lasecki, Christopher Miller, Adam Sadilek, Andrew Abumoussa, Donato Borrello, Raja Kushalnagar, and Jeffrey Bigham. 2012. Real-time captioning by groups of non-experts. In Proceedings User interface software and technology (UIST '12). ACM, NY, USA, 23-34. DOI: https://doi.org/10.1145/2380116.2380122

43. Jon P. Barker, Ricard Marxer, Emmanuel Vincent, Shinji Watanabe. 2017. The CHiME challenges: Robust speech recognition in everyday environments. New Era for Robust Speech Recognition. Springer, Cham, 327-344. 